\title{
Analysis of polaron effects in the cyclotron resonance of $n$-GaAs and AlGaAs-GaAs heterojunctions
}

\author{
H. Sigg and P. Wyder \\ Physics Laboratory and Research Institute for Materials, University of Nijmegen, \\ Toernooiveld, 6525 ED Nijmegen, The Netherlands
}

\author{
J. A. A. J. Perenboom \\ High Field Magnet Laboratory, University of Nijmegen, Toernooiveld, 6525 ED Nijmegen, The Netherlands
}

(Received 3 December 1984)

\begin{abstract}
Cyclotron resonance (CR) was measured at high magnetic fields in three-dimensional bulk $n$ GaAs and in the two-dimensional (2D) electron system of an AlGaAs-GaAs heterojunction, at a number of far-infrared laser lines, up to energies close to the longitudinal optical phonon energy, $\hbar \omega_{\text {LO }}$. A quantitative analysis of the CR as a function of magnetic field is presented, taking into account dimensionality, band nonparabolicity, and polaron effects. It is found that screening is important in 2D systems, and from an analysis of polaron effects in the CR of the AlGaAs-GaAs heterojunction, a value for the screening strength in a $2 \mathrm{D}$ electron system is obtained. Our analysis of data very close to resonance with the optical phonon shows that band nonparabolicity has to be included explicitly in the calculation of polaron corrections to obtain the proper asymptotic limit $\hbar \omega_{\mathrm{LO}}$ for the CR transition energy.
\end{abstract}

\section{INTRODUCTION}

For many years, the measurement of polaron effects on the cyclotron resonance (CR) has been an excellent tool for studying the electron-phonon interaction in pure polar semiconductors of high carrier mobility. Numerous CR investigations have been done in bulk [three-dimensional (3D)] semiconductors, and it is generally accepted that the CR effective mass is enhanced due to polaron effects, i.e., due to the virtual coupling of a quasifree electron with the optical phonon; the enhancement is most prominent in the resonant regime close to the energy of the optical phonon $\hbar \omega_{\text {LO }}{ }^{1,2}$

Recently, very pure semiconductor systems have become available with interfaces that support a quasi-twodimensional (2D) electron gas of very high mobility. These systems include heterojunctions, superlattices with multiple interfaces, and metal-oxide-semiconductor fieldeffect transistors (MOSFET's), and they are of great technological importance as components for optoelectronic devices and fast switches. There is also a growing interest in fundamental properties of the $2 \mathrm{D}$ electron gas, such as the electron-electron and electron-phonon interactions, and the influence of screening and localization. ${ }^{3} \mathrm{CR}$ measurements were therefore soon applied to study polaron effects in these $2 \mathrm{D}$ systems as well. ${ }^{4}$

To date, the knowledge and understanding of the 2D polaron effect is still much less well developed than for the $3 \mathrm{D}$ case. Horst, Merkt, and Kotthaus ${ }^{5}$ studied the polaron effect on $\mathrm{CR}$ in an InSb space-charge layer and found, qualitatively just as in bulk InSb, an anomaly in the CR effective mass at about the energy of the optical phonon. Seidenbusch, Lindemann, Lassnig, Edlinger, and Gornik $^{6}$ studied the polaron effect in AlGaAs-GaAs and found no polaron mass enhancement in marked contrast with bulk GaAs, where a substantial mass enhancement due to polaron effects is found. ${ }^{7,8}$ This is in clear contrast with theoretical predictions that the $2 \mathrm{D}$ polaron mass enhancement should be even larger than for the 3D case. Very recently, it was suggested that the $2 \mathrm{D}$ mass enhancement may be quenched by screening and the finite extension of the quasi-two-dimensional electron wave functions, ${ }^{9}$ or that the polaron effect is weakened due to a phonon inactive interface layer. ${ }^{10}$

In this paper we will present measurements of the CR in $n$-GaAs and in AlGaAs-GaAs heterojunctions up to energies close to $\hbar \omega_{\mathrm{LO}}$. We will give an analysis of the polaron effects on the $3 \mathrm{D}$ and $2 \mathrm{D} \mathrm{CR}$ data, and will discuss the dimensionality dependence of these effects for conduction-band electrons in $n$-GaAs (3D) and in AlGaAs-GaAs (2D), respectively.

The details of samples and measurement technique are summarized in Sec. II. The calculation of polaron corrections is discussed in Sec. III, based on a second-order perturbation analysis of the Fröhlich Hamiltonian. It is shown how dimensionality, band nonparabolicity and screening enters in these calculations. Finally, in Sec. IV, we will analyze quantitatively the measured cyclotron resonance energies and we will show that screening is important in a $2 \mathrm{D}$ electron gas, and that the resonant polaron contributions in 3D $n$-GaAs and 2D AlGaAs-GaAs are quite similar.

\section{EXPERIMENTAL DETAILS}

We have measured the transmission and photoconductivity of $n$-GaAs and AlGaAs-GaAs heterojunctions in 
high magnetic fields and at far-infrared (FIR) wavelengths in a conventional way. The radiation was generated by a FIR molecular laser, pumped with a $\mathrm{CO}_{2}$ laser; the radiation was coupled to the sample in the Faraday configuration with the Poynting vector parallel to the applied magnetic field. The laser system was optimized for the generation of short-wavelength FIR laser lines, as we have described elsewhere, ${ }^{11}$ so that the CR in GaAs could be measured at energies close to the optical-phonon energy $\hbar \omega_{\text {LO }}=36.75 \mathrm{meV}^{12}$ The samples were mounted in a continuous-flow cryostat or immersed in liquid helium in the bore of a high-field hybrid magnet system. ${ }^{13}$ The FIR laser radiation was guided into the cryostat by oversized light pipes and coupled to the sample with a cone. The transmitted radiation was measured with a helium-cooled Ge bolometer in a second cryostat at some distance from the magnetic field.

The very pure epitaxially grown $n$-GaAs sample had an electron density at $77 \mathrm{~K}$ of $n_{e}=4.4 \times 10^{14} \mathrm{~cm}^{-3}$ and a mobility $\mu=67000 \mathrm{~cm}^{2} / \mathrm{V} \mathrm{s}$, deduced from Hall resistance and resistivity measurements. The transmission of this sample was measured at $T \simeq 10 \mathrm{~K}$; unfortunately, due to reststrahlen reflection, the signal becomes too weak to be observed for energies above $30 \mathrm{meV}$. For measuring photoconductivity, the optimum temperature was found to be $T \simeq 15 \mathrm{~K}$, and $\mathrm{CR}$ was observed with this technique over the whole range of energies. Figure 1(a) shows the photoconductivity at $h v=32.76 \mathrm{meV}$ as a function of the magnetic field; here the splitting of the CR signal due to spin-up and spin-down Landau levels is very well resolved. The spin-split peaks are so narrow that the midfield value of the transition can be determined with a rela-

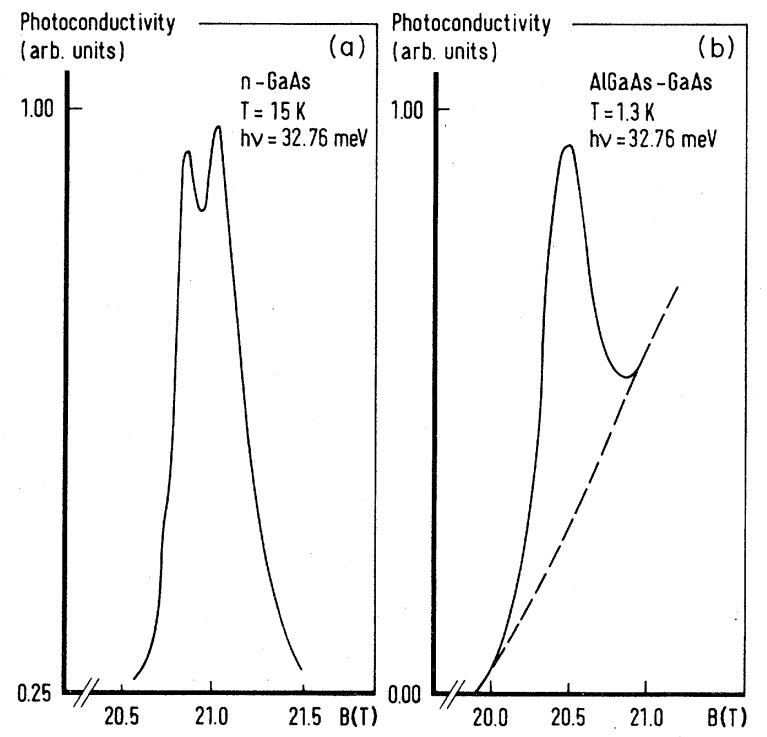

FIG. 1. Photoconductivity as a function of magnetic field at an energy of incident radiation of $h v=32.76 \mathrm{meV}$. (a) For $n$ GaAs measured at $T=15 \mathrm{~K}$ (note the very well resolved spin-up and spin-down resonances); (b) for an AlGaAs-GaAs heterojunction, measured at $T=1.3 \mathrm{~K}$; the dashed curve indicates the background signal. tive uncertainty of $\Delta B / B \simeq 10^{-3}$.

The $\mathrm{Al}_{x} \mathrm{Ga}_{1-x}$ As-GaAs $(x=0.3)$ heterojunctions were grown by means of metal organic chemical vapor phase deposition. The electron density was $n_{e}=4.0 \times 10^{11}$ $\mathrm{cm}^{-2}$, and the mobility was $\mu=30000 \mathrm{~cm}^{2} / \mathrm{V} \mathrm{s}$, deduced from Shubnikov-de Haas oscillations in the resistance and the resistivity. The transmission measurements were done on a sample with Corbino geometry and the photoconductivity measurements on a sample shaped as a standard Hall strip, with the sample at $T=1.3 \mathrm{~K}$. The $2 \mathrm{D}$ samples were oriented with the interface perpendicular to the direction of the magnetic field. As in the bulk case, reststrahlen reflection prevents transmission measurements at the highest energies. Figure 1(b) shows the photoconductivity of the AlGaAs-GaAs heterojunction, again at $h v=32.76 \mathrm{meV}$, indicating the cyclotron resonance which is superposed on a strong background signal. The background photoconductivity is related to the strong temperature dependence of the Hall resistance in the presence of the quantum Hall effect, ${ }^{14}$ and using measurements at $T=6 \mathrm{~K}$ we could evaluate the background signal and subtract it from the photoconductivity at lower temperatures.

In Fig. 2 and Table I we present our experimental

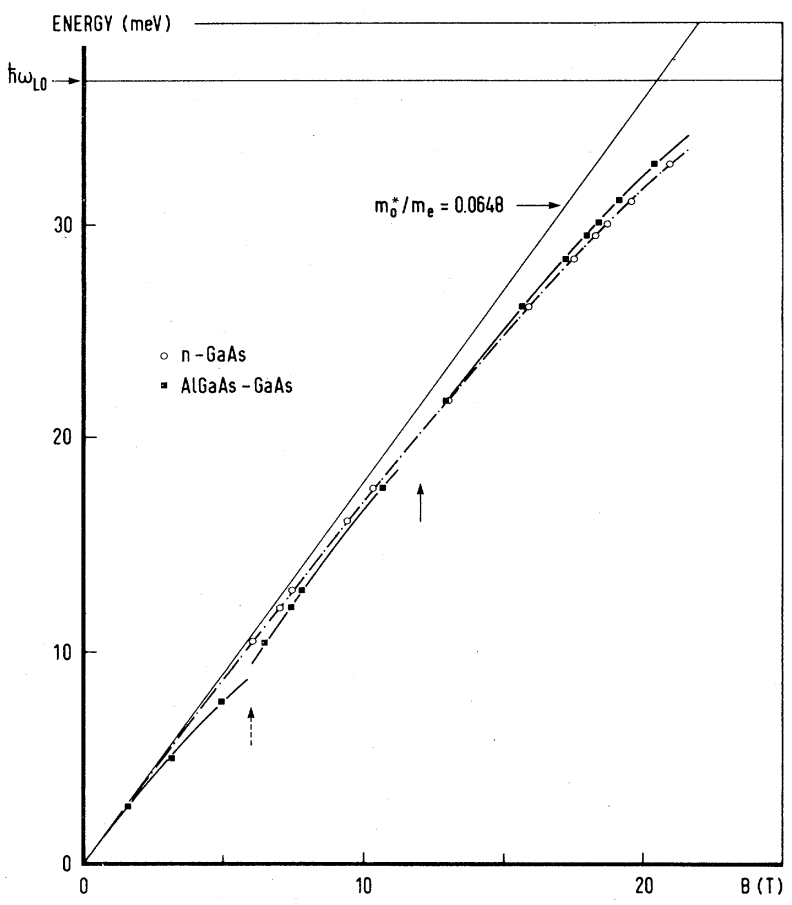

FIG. 2. Observed CR transition energy as a function of magnetic field in 3D $n$-GaAs (O) and 2D AlGaAs-GaAs heterojunctions ( $)$. The dot-dash curve through the 3D data and solid curve through the $2 \mathrm{D}$ data are to guide the eye. The deviation from the zero-order transition energy $\hbar \omega_{c}^{0}=e \hbar B / m_{0}^{*}$ (straight line), is due to band nonparabolicity and to polaron effects: mass enhancement and hybridization for CR energies close to the optical-phonon energy $\hbar \omega_{\text {LO }}$. The discontinuity indicated with a solid arrow is tentatively ascribed to filling up of Landau levels, the discontinuity with a dashed arrow to a plasma anomaly. 
TABLE I. Experimental data.

\begin{tabular}{ccc}
\hline \hline $\begin{array}{c}\text { Energy } \\
h v(\mathrm{meV})\end{array}$ & \multicolumn{2}{c}{ Resonance field $\boldsymbol{B}(\mathrm{T})$} \\
AlGaAs-GaAs
\end{tabular}

values of the magnetic field $B$ and of the FIR energy $\epsilon=h v$ at the CR. For both $n$-GaAs and AlGaAs-GaAs heterojunctions the transition energy between the Landau levels increases with the magnetic field, and with increasing field the CR transition energy becomes more and more reduced below $\hbar \omega_{c}^{0}$, i.e., the curve bends increasingly stronger away from the straight line representing the zero-order transition energy $\hbar \omega_{c}^{0}=e \hbar B / m_{0}^{*}$. For $B \rightarrow \infty$ the CR energy seems to approach the energy of the optical phonon $\hbar \omega_{\text {LO }}$, as one expects for resonant polaron effects. The data of the AlGaAs-GaAs heterojunctions reveal two anomalies in the low-field regime where the $2 \mathrm{D} \mathrm{CR}$ is not a smooth function of the magnetic field as the CR of $n$ GaAs. In this paper we will be mainly concerned with polaron effects which are most prominent near resonance with the optical phonon, at high fields.

\section{THEORETICAL ANALYSIS}

\section{A. Polarons}

For both the $2 \mathrm{D}$ and $3 \mathrm{D}$ cases, the polaron model is analyzed in terms of second-order perturbation theory of the Fröhlich Hamiltonian. The dimensionality dependence will then enter through the appropriate zero-order energy dispersion relation and the appropriate electron wave function. On the other hand, however, the phonon system is assumed to be bulk like in the 2D case as well, and in our estimates we take the bulk value for the optical-phonon energy $\hbar \omega_{\mathrm{LO}}$ and for the coupling constant $\alpha$. When the conduction band is not parabolic, corrections due to band nonparabolicity (BNP) have to be included in the zero-order energy levels. BNP will be discussed in more detail in Sec. III B.

When a virtual coupling of the conduction electrons with the optical phonon is included, the energies of the Landau levels are given in the weak coupling limit by ${ }^{15}$

$$
E_{f}^{\mathrm{pol}}=E_{f}^{0}+\sum_{i, \mathrm{q}} v_{q}^{2} \frac{|M|_{i f}^{2}}{E_{f}^{\mathrm{pol}}-E_{i}^{0}-\hbar \omega_{\mathrm{LO}}},
$$

with

$$
v_{q}^{2}=\frac{4 \pi \alpha \hbar\left(\hbar \omega_{\mathrm{LO}}\right)^{3 / 2}}{\left(2 m_{0}^{*}\right)^{1 / 2} \Omega q^{2}}
$$

and

$$
|M|_{i f}^{2}=\left|\int_{\Omega} \Psi_{f}^{*}(\mathbf{r}) e^{i \mathbf{q} \cdot r} \Psi_{i}(\mathbf{r}) d^{3} r\right|^{2},
$$

where $E_{i}^{0}, E_{f}^{0}, \Psi_{i}(\mathbf{r})$, and $\Psi_{f}(\mathbf{r})$ are, respectively, the electron energies and wave functions of the unperturbed (zero-order) electrons in the intermediate and final Landau state, and $\Omega$ is the volume of the crystal. Equation (1) describes the energy spectrum of the free-electron system as a function of the magnetic field, when its electron charge distribution $|\Psi(\mathbf{r})|^{2}$ is weakly coupled to the polarization field of the optical phonon; the strength of this coupling is given by $v_{q}^{2}|M|^{2}$ which is proportional to the Fröhlich coupling constant $\alpha$. A summation has to be carried out over the phonon wave vector $\mathbf{q}$ and all intermediate states $i=\left(n, k_{\|}\right)$, where $n$ is the Landau-level index and $k_{\|}$the electron wave vector in the direction parallel to the quantizing magnetic field.

In Eq. (1) the following approximations are made: the energy of the optical phonon is assumed to be independent of the phonon wave vector $q$, only single-phonon scattering processes are considered, and only virtual phonons are included in the calculation (at zero temperature, there are no phonons in the system). These approximations are valid for our experimental conditions: in GaAs, one has $\alpha \simeq 0.065<<1$, and the measurements were carried out at low temperatures $(T \simeq 10 \mathrm{~K}$ for $n$-GaAs, and $T \simeq 1 \mathrm{~K}$ for the AlGaAs-GaAs heterojunctions).

We will now analyze the interaction of a phonon with electrons in the following two cases: when the electrons are quasifree in all three dimensions with isotropic effective mass $m_{0}^{*}$, and when the electrons are quasifree in only two dimensions $\left(m_{x}^{*}=m_{y}^{*}=m_{0}^{*}\right)$, confined in the $z$ direction parallel to the magnetic field by some interface potential. (We think of a 3D polaron as a bulk electron interacting with a bulk phonon, and a $2 \mathrm{D}$ polaron as a confined electron interacting with a bulk phonon as well.) In our model the dimensionality of the systems enters through the electron wave function in the matrix elements $|M|_{i f}^{2}$, and through the $k_{\|}$dependence of the zero-order electron energy $E^{0}$. For a quasifree bulk electron, in a magnetic field along the $z$ direction and in the $n$th Landau level, the energy and wave function are given, respectively, by

$$
\begin{aligned}
& E_{n k_{\|}}(B)=\left(n+\frac{1}{2}\right) \hbar \omega_{c}^{0}+\frac{\hbar^{2} k_{\|}^{2}}{2 m_{0}^{*}}, \\
& \Psi_{n k_{\|}}(\mathbf{r})=c \Phi_{n}(x, y) e^{i k_{\| !} z},
\end{aligned}
$$

where $\hbar \omega_{c}^{0}=e \hbar B / m_{0}^{*}$ is the cyclotron resonance energy, $c$ is a normalization factor, and $\Phi_{n}$ the $n$th harmonic oscillator wave function. For a confined $2 \mathrm{D}$ electron gas, one has

$$
\begin{aligned}
& E_{n s}(B)=\left(n+\frac{1}{2}\right) \hbar \omega_{c}^{0}+E_{s}, \\
& \Psi_{n s}(\mathbf{r})=c^{\prime} \Phi_{n}(x, y) f_{s}(z),
\end{aligned}
$$


where $E_{s}$ is the energy of the $s$ th subband in the confining potential well, $c^{\prime}$ is a normalization factor, and $\left|f_{s}(z)\right|^{2}$ determines the extension of the wave function of the $s$ th subband in the $z$ direction; in the ideal 2D case $|f(z)|^{2}=\delta(z)$. The summation in Eq. (1a) has to be carried out over all intermediate states $i=\left(n, k_{\|}\right)$for the 3D case, substituting Eqs. (2a) and (2b) where appropriate, and over $i=(n, s)$ and substituting Eqs. (3a) and (3b) in the $2 \mathrm{D}$ case. As a result, one gets directly from Eq. (1) for the change in transition energy between Landau levels due to the polaron effect: $\Delta \epsilon^{\mathrm{pol}}=E_{1}^{\mathrm{pol}}-E_{0}^{\mathrm{pol}}-\hbar \omega_{c}^{0}$. The polaron mass enhancement $\Delta=1-m_{0}^{*} / m_{\text {pol }}^{*}$ is given by $\Delta \epsilon^{\mathrm{pol}} / \hbar \omega_{c}^{0}$. In the limit of low fields, the calculation of $\Delta \epsilon^{\mathrm{pol}}$ requires a summation over the whole range of Landau levels, but in the final result, only terms linear with the magnetic field need to be considered. For the mass enhancement in this low-field limit one obtains in the 3D case $\Delta=\frac{1}{6} \alpha$ (Ref. 15 ) and in the $2 \mathrm{D}$ case $\Delta=\frac{1}{8} \pi \alpha .{ }^{9,16}$ In the resonant or high-field limit, we need to consider only that single term in the summation which can become resonant: for a final state $f=(1,0)$ only the intermediate state $i=\left(0, k_{\|}\right)$in the $3 \mathrm{D}$ case, or the state $i=(0,0)$ in the $2 \mathrm{D}$ case. For $\hbar \omega_{c}^{0} / \hbar \omega_{\mathrm{LO}}=1$ one then obtains $\Delta=\left(\frac{1}{2} \alpha\right)^{2 / 3}$ (Refs. 15 and 17) for the $3 \mathrm{D}$ case, and $\Delta=\left(\frac{1}{4} \alpha \sqrt{\pi}\right)^{1 / 2}$ (Ref. 18) for the 2D case.

From this analysis it follows that the mass enhancement in a 2D AlGaAs-GaAs heterojunction is about twice as large as in bulk GaAs, and largest in the low-field regime. However, we will show below that the mass enhancement in a 2D system will be quenched considerably by effects due to screening and due to extension of the wave function in the direction perpendicular to the interface.

\section{B. Band nonparabolicity}

The band nonparabolicity (BNP) corrections to the free-electron energy can be calculated from a two-band $\mathbf{k} \cdot \mathbf{p}$ approximation, where the coupling is considered between one conduction band and one valence band separated by an energy gap $\epsilon_{g}$. This sort of calculation basically leads to a BNP corrected conduction-band energy $E^{\mathrm{BNP}}=E^{0}\left(1+E^{0} / \epsilon_{g}\right)$, where $E^{0}$ is the energy of the quasifree electron, given by the Eqs. (2a) and (3a). The transition energy from the $(n-1)$ th to the $n$th Landau level, including the nonparabolicity of the conduction band, is given by

$$
\hbar \omega_{c}^{0}+\Delta \epsilon^{\mathrm{BNP}} \simeq \hbar \omega_{c}^{0}\left(1-\frac{2 n \hbar \omega_{c}^{0}}{\epsilon_{g}}-\frac{2 K}{\epsilon_{g}}\right)
$$

where in the $3 \mathrm{D}$ case, the kinetic energy $K$ is $\hbar^{2} k_{\|}^{2} / 2 m_{0}^{*}$. For the case of AlGaAs-GaAs, the BNP corrections have been evaluated using a more sophisticated $\mathbf{k} \cdot \mathbf{p}$ model, ${ }^{19}$ where the 2D potential well confining the conduction electrons is included explicitly in the Hamiltonian. In the limit of large gap $\epsilon_{g}$, the same expression as Eq. (4) is obtained, but now the expectation value of the kinetic energy in the $z$ direction has to be substituted for $K$ and not the total subband energy as one would expect from the more simple arguments given above.

For a nondegenerate $3 \mathrm{D}$ system in the quantum limit, there are electrons only in the lowest Landau level and $k_{\|} \simeq 0$, and transitions between the levels $n=0 \rightarrow 1$ only contribute to Eq. (4). For a degenerate 2D system, however, the lowest unoccupied Landau level is just above the Fermi energy $\left(n \hbar \omega_{c}^{0} \simeq E_{F}\right)$, and consequently, in the lowfield limit, the BNP correction remains significant and does not tend to zero as in the 3D case. Therefore, at low fields a mass enhancement in a $2 \mathrm{D}$ system is expected when compared with the bulk material.

\section{Screening in a 2D electron system}

Screening enters the calculation of polaron effects through $v_{q}^{2}$ in Eq. (1). In a Thomas-Fermi approximation, for a $3 \mathrm{D}$ electron-gas screening decreases with decreasing electron density, and consequently it can be neglected for low density, nondegenerate semiconductors at low temperatures.

For a 2D electron gas Stern and Howard ${ }^{20}$ have calculated the screening of the Coulomb potential of a point charge in the Thomas-Fermi approximation. The longitudinal polarization field of the optical photon can be expressed in terms of an effective charge distribution, proportional to $q \exp (i \mathbf{q} \cdot \mathbf{r})$; we then obtain $v_{\mathbf{q}}^{2 \mathrm{D}}=v_{q} q_{\perp} /$ $\left(q_{\perp}+q_{\mathrm{TF}}\right)$, where $v_{q}$ is as defined in Eq. (1b); $q_{\perp}$ is the phonon wave vector perpendicular to the magnetic field, i.e., parallel to the interface, and $q_{\mathrm{TF}}$ is the 2D ThomasFermi screening wave vector $q_{\mathrm{TF}}=m_{0}^{*} e^{2} / 2 \pi \epsilon_{0} \kappa \hbar^{2}$, where $\kappa$ is the bulk dielectric constant. This result is valid for an ideally restricted 2D electron gas only. For the case, where $\left|f_{0}(z)\right|^{2} \neq \delta(z)$ we find, in agreement with a calculation by Price, ${ }^{21}$

$$
v_{\mathbf{q}}^{2 \mathrm{D}}=v_{q} \frac{q_{\perp}}{q_{\perp}+q_{\mathrm{TF}} \eta\left(q_{\perp}\right)}
$$

where

$$
\eta\left(q_{\perp}\right)=\int_{-\infty}^{+\infty} \frac{2 q_{\perp}}{\left(\xi^{2}+q_{\perp}^{2}\right)}|\rho(\xi)|^{2} d \xi .
$$

$\rho(\xi)$ is the Fourier transform of $\left|f_{0}(z)\right|^{2}$. The screening is strongly anisotropic, dependent on the component of the wave vector of the optical phonon parallel to the interface and on the spatial extension of the electron wave function.

\section{ANALYSIS OF THE EXPERIMENTAL RESULTS}

\section{A. Polaron effects in 3D: the case of $n-G a A s$}

The results of the cyclotron resonance experiments in bulk $n$-GaAs are presented in Fig. 2 and Table I. As can be seen in the figure, at low fields the Landau-level transition energy increases almost linearly with the magnetic field; at high fields, as one approaches the energy of the optical phonon, the effective mass becomes more and more enhanced. Note that the contribution to the mass enhancement due to polarons cannot simply be deduced from the data, but band nonparabolicity effects must be taken carefully into account first. In this section we will give a detailed description of the analysis of polaron effects in bulk $n$-GaAs. 
There is a considerable amount of literature on the low-field regime. Recently, Das Sarma and Mason have developed a theory to describe polaron effects in the lowfield limit for the case of a nonparabolic band, as, e.g., in $n-\mathrm{GaAs} ;{ }^{22}$ they calculated the change of the CR transition energy due to polaron effects for a system with nonparabolic zero-order energy band in a Rayleigh-Schrödinger perturbation model (RSPT), and we will denote this contribution by $\Delta \epsilon^{\mathrm{RSPT}}$. The most intriguing outcome of their calculation is the fact that BNP leads to an enhancement of polaron effects, and that therefore it is not sufficient to account for BNP merely by a shift of the conduction-band energy level with respect to the parabolic case. Accordingly, we will follow Das Sarma and Mason, who carefully included BNP effects in the evaluation of polaron corrections.

An expression for the change of the transition energy due to $\mathrm{BNP}$ of the conduction band $\Delta \epsilon^{\mathrm{BNP}}$ is given in Eq. (4). Das Sarma and Mason obtained the following result for $\Delta \epsilon^{\mathrm{RSPT}}:^{22}$

$$
\Delta \epsilon^{\mathrm{RSPT}}=-\hbar \omega_{c}^{0}\left[1.23 \frac{\alpha}{6}+1.05 \frac{3 \alpha}{20} \frac{\hbar \omega_{c}^{0}}{\hbar \omega_{\mathrm{LO}}}\right],
$$

where the numerical factors 1.23 and 1.05 in the realistic case of bulk GaAs with a nonparabolic band are the numbers found for the enhancement over the case of a simple parabolic band.

In Fig. 3 we plotted our experimental CR data $\left(\epsilon-\hbar \omega_{c}^{0}\right) / \hbar \omega_{\text {LO }}$ as a function of $\hbar \omega_{c}^{0} / \hbar \omega_{\text {LO }}$. The lower en-

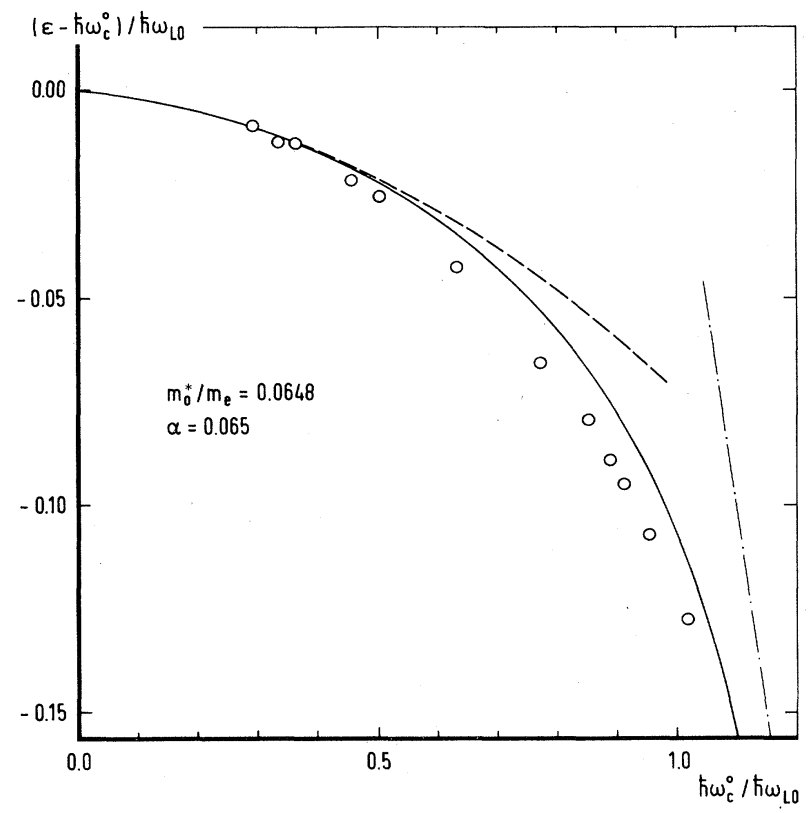

FIG. 3. Measured deviation of the CR energy from the zeroorder transition energy $\left(\epsilon-\hbar \omega_{c}^{0}\right) / \hbar \omega_{\text {LO }}$ as a function of $\hbar \omega_{c}^{0} / \hbar \omega_{\text {LO }}$ for bulk $n$-GaAs. The experimental data are represented by $(O)$, the solid curve is calculated using the effective mass and coupling constant determined from the low-field data, and the dashed curve represents the low-field limit $\Delta \epsilon^{\mathrm{BNP}}+\Delta \epsilon^{\mathrm{RSPT}}$. The dot-dashed line gives the proper asymptotic limit $\left(1-\hbar \omega_{c}^{0} / \hbar \omega_{\mathrm{LO}}\right)$. ergy range, below and around our lowest CR data on $n$ $\mathrm{GaAs}$, is well covered by the CR measurements of Lindemann, Lassnig, Seidenbusch, and Gornik, ${ }^{7}$ and the data in our study measured at the same laser wavelengths coincide within the experimental accuracy. The result of Eqs. (4) and (6) can be compared with all of these low-field data and a good fit can be obtained using a value for the effective mass of $m_{0}^{*}=0.0648 m_{e}$ and for the coupling constant of $\alpha=0.065$. Note that often too large a value of $\alpha$ is found from polaron measurements; ${ }^{22}$ the value of $\alpha$ now obtained agrees very well with the value $0.065 \pm 0.005$ determined by dielectric measurements. ${ }^{23,24}$ The theoretical result of $\Delta \epsilon^{\mathrm{BNP}}+\Delta \epsilon^{\mathrm{RSPT}}$ is indicated by the dashed curve in Fig. 3. As would be expected, this low-field approximation does not describe the data at high fields, close to resonance with the optical phonon very well. Due to the hybridization, the CR transition energy for the lower branch will asymptotically approach $\hbar \omega_{\text {LO }}$ for high magnetic fields; the appropriate limit for the data of Fig. $3\left(1-\hbar \omega_{c}^{0} / \hbar \omega_{\text {LO }}\right)$ is indicated by the dot-dashed line.

A careful analysis of Eq. (1) shows that in the low-field limit a summation over a large range of Landau levels has to be carried out, but near resonance only one term will be dominating-the coupling of the $n$th Landau level with the intermediate state $n-1$, when $\hbar \omega_{c}^{0} \simeq \hbar \omega_{\text {LO }}$. Accordingly, Larsen ${ }^{15}$ obtains in the resonant limit the following implicit equation for the polaron correction to the transition energy:

$\frac{\Delta \epsilon^{\mathrm{pol}}}{\hbar \omega_{\mathrm{LO}}}=-\frac{\alpha}{2}\left[1-\lambda^{2}-\frac{\Delta \epsilon^{\mathrm{pol}}}{\hbar \omega_{\mathrm{LO}}}\right)^{-1 / 2}+g\left(\frac{\hbar \omega_{c}^{0}}{\hbar \omega_{\mathrm{LO}}}\right)$.

Larsen ${ }^{15}$ considered the case of a parabolic band, then $\lambda^{2}=\hbar \omega_{c}^{0} / \hbar \omega_{\text {LO }}$ leading to a linear increase of $\lambda^{2}$ with the magnetic field; he also chose the function $g$ to be zero. In the case of a nonparabolic band, as in $n$-GaAs, it is reasonable to substitute $\lambda^{2}=\left(\hbar \omega_{c}^{0}+\Delta \epsilon^{\mathrm{BNP}}\right) / \hbar \omega_{\mathrm{LO}}$; for high magnetic fields $\Delta \epsilon^{\mathrm{pol}}$ will then tend to $\hbar \omega_{\mathrm{LO}}\left(1-\lambda^{2}\right)$ $=\hbar \omega_{\mathrm{LO}}-\hbar \omega_{c}^{0}-\Delta \epsilon^{\mathrm{BNP}}$. At low fields, however, $\Delta \epsilon^{\mathrm{pol}}$ should approach the result $\Delta \epsilon^{\mathrm{RSPT}} \cdot{ }^{22}$ This is achieved if we substitute in Eq. (7)

$$
g\left(\hbar \omega_{c}^{0} / \hbar \omega_{\mathrm{LO}}\right)=\Delta \epsilon^{\mathrm{RSPT}}+g^{\prime}\left(\hbar \omega_{c}^{0} / \hbar \omega_{\mathrm{LO}}\right),
$$

with

$$
g^{\prime}(x)=\frac{1}{2} \alpha+\frac{1}{4} \alpha x+\frac{3}{16} \alpha x^{2} .
$$

At low fields $\Delta \epsilon^{\mathrm{pol}}$ will then approach $\Delta \epsilon^{\mathrm{RSPT}}$ of Eq. (6). Using Eq. (7) in this way we have evaluated $\Delta \epsilon^{\text {pol }}$ over the whole range of fields. The result of $\Delta \epsilon^{\mathrm{pol}}+\Delta \epsilon^{\mathrm{BNP}}$ is shown in Fig. 3 as a solid curve. As can be seen, this crude approximation describes the data remarkably well. It is noteworthy, however, that a larger value for $\alpha$ would result in a better agreement of the present theory with the data at higher magnetic fields; such an effective increase of $\alpha$ at high fields may be brought about by a more careful analysis (along the lines of Das Sarma and Mason ${ }^{22}$ ) of the single resonant term of Eq. (1) used to derive the high-field expression Eq. (7).

When, as is usually done, BNP effects on the polaron contributions are neglected and the analysis discussed above is carried through, a result is obtained which can 
describe the data for $\hbar \omega_{c}^{0} / \hbar \omega_{\text {LO }}<0.85$ quite well [however, leading to larger values of the parameters, i.e., $\alpha=0.08$ and $m_{0}^{*}=0.0650 m_{e}$ (Ref. 7)], but the discrepancy becomes dramatically larger as the field increases. This is due to the fact that in this model, the CR transition energy now does not approach the correct limit but $\hbar \omega_{\mathrm{LO}}+\Delta \epsilon^{\mathrm{BNP}}$, and the theoretical curve bends much stronger than the experimental data. To assure that the transition energy has the proper asymptotic limit $\hbar \omega_{\text {LO }}$ it is therefore essential to carry BNP through the calculations of Eq. (1) for the polaron effects.

\section{B. Polaron effects in 2D: the case of AlGaAs-GaAs}

\section{Screening and wave-function extension}

As discussed in Sec. III C, screening is not important for a low-density electron gas as in the case of nondegenerate bulk $n$-GaAs. But we will show that it may become an important effect for a 2D electron gas as in the case of an AlGaAs-GaAs heterojunction. Until now, in most models of polaron effects in $2 \mathrm{D}$ systems screening was not included. ${ }^{19,25}$

Basically, effects of screening on the polaron mass enhancement can be calculated from Eqs. (1) and (5). For the evaluation, the $z$ dependence of the wave function is needed. A convenient expression is given by $\operatorname{Stern}^{26}$ in the form of

$$
f_{0}(z, \beta)=(1 / \sqrt{2})\left(\beta q_{0}\right)^{3 / 2} z \exp \left(-\beta q_{0} z / 2\right) .
$$

$\beta$ is a parameter that scales the extension of the wave function in the $z$ direction and $q_{0}=\left(2 m_{0}^{*} \omega_{\mathrm{LO}} / \hbar\right)^{1 / 2}$; the ideal 2D limit is obtained when $\beta \rightarrow \infty$. We will also introduce a scaling factor $\gamma$ for the strength of the screening by defining an effective screening wave vector $\gamma \gamma_{0} q_{0}$, where $\gamma_{0}=q_{\mathrm{TF}} / q_{0}$. With these definitions, one can again derive the polaron mass enhancement $\Delta=\Delta \epsilon^{\mathrm{pol}} / \hbar \omega_{c}^{0}$ and the following results are obtained for the two limiting cases: in the low-field limit

$$
\begin{aligned}
& \Delta\left[\beta, \gamma, \frac{\hbar \omega_{c}^{0}}{\hbar \omega_{\mathrm{LO}}} \rightarrow 0\right. \\
&=2 \alpha \int_{0}^{\infty} \frac{x^{4}}{\left(1+x^{2}\right)^{3}} \frac{\eta(x / \beta)}{\left[x+\eta(x / \beta) \gamma_{0} \gamma\right]^{2}} d x
\end{aligned}
$$

and in the resonant limit for $\hbar \omega_{c}^{0} / \hbar \omega_{\mathrm{LO}}=1$

$$
\begin{aligned}
& \Delta\left[\beta, \gamma, \frac{\hbar \omega_{c}^{0}}{\hbar \omega_{\mathrm{LO}}}=1\right] \\
& \quad=\sqrt{\alpha}\left[\int_{0}^{\infty} x^{4} e^{-x^{2}} \frac{\eta(x / \beta)}{\left[x+\eta(x / \beta) \gamma_{0} \gamma\right]^{2}} d x\right]^{1 / 2}
\end{aligned}
$$

where from Eq. (5), $\eta(\xi)=\left(8+9 \xi+3 \xi^{2}\right) / 8(1+\xi)^{3}$. A similar calculation was done in the low-field limit by Das Sarma, ${ }^{9}$ where ideal 2D screening was considered. The result of Das Sarma is qualitatively similar but the mass enhancement calculated as a function of $\beta$ is systematically larger than our result; note that Das Sarma uses a slightly different definition for $v_{\mathrm{q}}^{2 \mathrm{D}}$ than our Eq. (5a). When the two expressions of Eq. (10) are evaluated with $\beta \rightarrow \infty$ and $\gamma \rightarrow 0$, the unscreened, ideal $2 \mathrm{D}$ result is again, for low fields, $\Delta(\infty, 0)=\frac{1}{8} \pi \alpha$, and for $\hbar \omega_{c}^{0} / \hbar \omega_{\mathrm{LO}}=1$, $\Delta(\infty, 0)=\left(\frac{1}{4} \alpha \sqrt{\pi}\right)^{1 / 2}$.

Figure 4 shows the polaron mass enhancement calculated with screening strength $\gamma$ and as a function of the wave-function extension parameter $\beta$, and normalized by the mass enhancement in the ideal $2 \mathrm{D}$ unscreened limit, both for the limit of low fields (dashed curves) and in the resonant limit for $\hbar \omega_{c}^{0} / \hbar \omega_{\mathrm{LO}}=1$ (solid curves). As one can see from the figure, the polaron mass enhancement is strongly quenched by screening, particularly in the lowfield limit, and also for low values of $\beta$, i.e., when the electrons are not strongly confined to the interface. The mass enhancement increases with $\beta$, but in the presence of screening it levels off at quite low values of $\beta$.

It is important to realize that the single subband model used in the present analysis will break down in the limits $\beta \rightarrow 0$ and $\beta \rightarrow \infty$. When $\beta$ is small, the energy differences between the lowest subbands become comparable to the thermal energy and the thermal population of the higher subbands has to be taken into account; a larger mass enhancement than calculated from Eq. (10) will then be found. Also, the mass enhancement is expected to approach the bulk value for $\beta \rightarrow 0$ rather than zero as shown in Fig. 4. On the other hand, when $\beta$ is large, both the

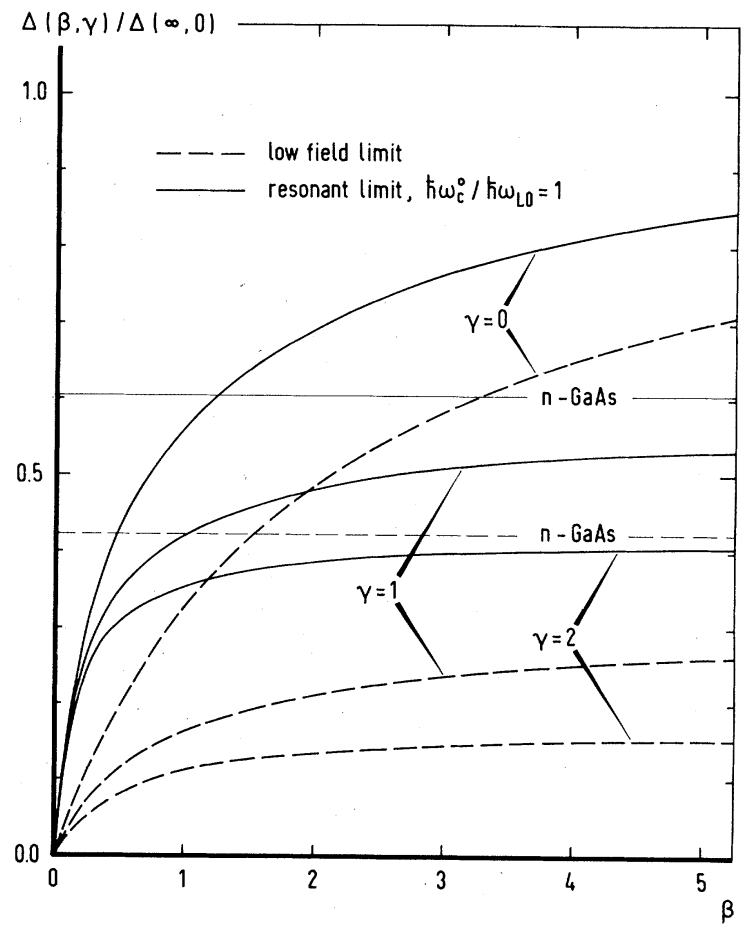

FIG. 4. Calculated polaron mass enhancement $\Delta=1-m_{0}^{*} / m_{\text {pol }}^{*}$ for a $2 \mathrm{D}$ system as a function of the wavefunction extension parameter $\beta$ and for values of the screening strength parameter $\gamma . \Delta(\beta, \gamma) / \Delta(\infty, 0)$, normalized to the mass enhancement of the ideal and unscreened $2 \mathrm{D}$ case, is evaluated for the low-field regime (dashed curves) and for the resonant polaron regime (solid curves). For comparison, the mass enhancement for bulk GaAs, calculated in these two limiting cases, is also shown. 
Fermi energy $\left(E_{F} \propto \beta^{3}\right)$ and the energies of the subbands $\left(E_{s} \propto \beta^{2}\right)$ become large, ${ }^{3}$ and consequently, for sufficiently large values of $\beta$, population of higher subbands has to be taken into account again. Also, in this case, the $z$ dependence of the screening should have been considered explicitly in our model of Eq. (5). For an AlGaAs-GaAs heterojunction at a temperature of about $2 \mathrm{~K}$, the single subband approximation should hold for $0.15<\beta<2.0$.

For a real AlGaAs-GaAs heterojunction, one has realistically $\beta \simeq 1$ and $\gamma \simeq 1$, and therefore Fig. 4 shows that the polaron mass enhancement will be reduced below the mass enhancement in bulk GaAs. In the low-field limit, the suppression of the mass enhancement is so strong that polaron effects will be very small. Near resonance, polaron effects are still important, but they depend strongly on the strength of screening.

\section{High-field data}

Because in the low-field limit the polaron effect is so strongly suppressed due to screening, it is a good approximation to consider only the single resonant term for the polaron correction in the summation of Eq. (1a),

$\Delta \epsilon^{\mathrm{pol}} \simeq \sum_{\mathrm{q}}\left[v_{\mathrm{q}}^{2 \mathrm{D}}(\beta, \gamma)\right]^{2} \frac{|M(\beta)|_{10}^{2}}{\hbar \omega_{c}^{0}+\Delta \epsilon^{\mathrm{BNP}}+\Delta \epsilon^{\mathrm{pol}}-\hbar \omega_{\mathrm{LO}}}$

This expression is similar to Eq. (7) in the sense that for high fields the transition energy between adjacent Landau levels $\hbar \omega_{c}^{0}+\Delta \epsilon^{\mathrm{BNP}}+\Delta \epsilon^{\mathrm{pol}}$ will approach the energy of the optical phonon.

Figure 5 shows the experimental values of $\epsilon-\hbar \omega_{c}^{0}$ together with the theoretical prediction $\Delta \epsilon^{\mathrm{BNP}}+\Delta \epsilon^{\mathrm{pol}}$ and the asymptotic limit $\hbar \omega_{\mathrm{LO}}-\hbar \omega_{c}^{0} . \Delta \epsilon^{\mathrm{BNP}}$ is calculated from Eq. (4) with kinetic energy $K=\frac{1}{4} \beta^{2} \hbar \omega_{\text {LO }}$ [using Stern's function for $f_{0}(z, \beta)$ (Ref. 26)] and with Landaulevel index $n=1$; for lower fields higher Landau levels seem to become important as we will discuss in more detail in the next paragraph. $\Delta \epsilon^{\text {pol }}$ is calculated from Eq. (11) where now $f_{0}(z, \beta)$ enters in the calculation of the matrix element $|M|^{2}$, so that this factor becomes dependent on $\beta$, and where $v_{\mathbf{q}}^{2 \mathrm{D}}(\beta, \gamma)$ is calculated from Eq. (5).

$\beta$ can be evaluated directly from the electron density; for our heterojunction, with $n_{e}=4.0 \times 10^{11} \mathrm{~cm}^{-2}$, we find $\beta=1.07$. The best fit of our model to the high-field data for $\beta=1.07$ is obtained with $\gamma=1.4$. This is indicated in Fig. 5 by the dashed curve. Note that this result suggests that screening is important, and stronger than expected from the Thomas-Fermi model. If we allow both parameters to be adjusted, a best fit is obtained with an even larger value for $\gamma(\gamma=2.1)$, and a consequently larger value for $\beta(\beta=1.3)$; the result indicated by a solid curve has a slightly weaker curvature and is in perfect agreement with the high-field data. We therefore conclude, that in order to describe accurately polaron effects in AlGaAs-GaAs heterojunctions near resonance with the optical phonon, screening has to be incorporated into the relevant model.

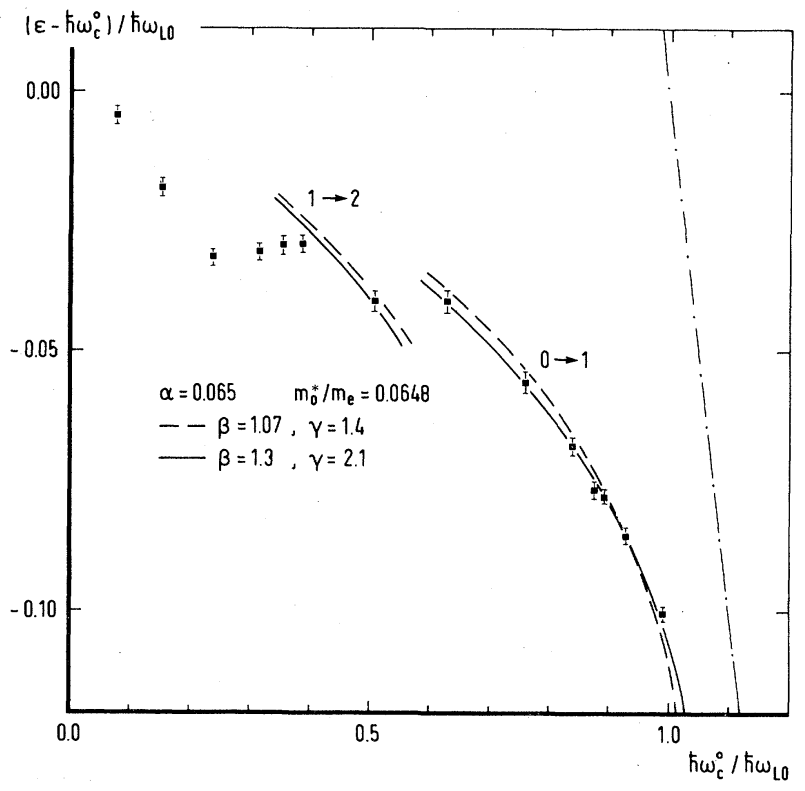

FIG. 5. Measured deviation of the CR energy from the zeroorder transition energy $\left(\epsilon-\hbar \omega_{c}^{0}\right) / \hbar \omega_{\mathrm{LO}}$ as a function of $\hbar \omega_{c}^{0} / \hbar \omega_{\text {LO }}$ for an AlGaAs-GaAs heterojunction. The experimental data are represented by $(\square)$ and the curves represent a best fit of the 2D polaron model with effective mass $m_{0}^{*} / m_{e}=0.0648$ and coupling constant $\alpha=0.065$. For the calculation at high fields, it was assumed that Landau-level transitions $0 \rightarrow 1$ take place, while at low fields, the $1 \rightarrow 2$ transition was taken into account. For the dashed curve, the wavefunction extension parameter was fixed at $\beta=1.07$, and the best fit was found for the screening strength $\gamma=1.4$; for the solid curve, a best fit for these two parameters is found at $\beta=1.3$ and $\gamma=2.1$. The dot-dashed line gives the proper asymptotic limit $\left(1-\hbar \omega_{c}^{0} / \hbar \omega_{\mathrm{LO}}\right)$.

\section{Discontinuities at lower energies}

At low fields two discontinuities in the CR as a function of energy seem to be present in the 2D AlGaAs$\mathrm{GaAs}$ heterojunction system (as also observed by other workers ${ }^{6,27}$, which are not described by the polaron given above. These discontinuities are marked by the arrows in Fig. 2.

From Figs. 2 and 5 it is clear that the 2D data below $\hbar \omega_{c}^{0} / \hbar \omega_{\mathrm{LO}} \simeq 0.6$ do not fit the general trend of the highfield data. Seidenbusch and co-workers ${ }^{6}$ studied CR in AlGaAs-GaAs heterojunctions with varying electron sheet density; they found a different effective mass in the highdensity and low-density regime. They could interpret the discontinuity as a transition from a regime, where Landau-level transitions $0 \rightarrow 1$ take place, to a regime, with Landau-level transitions $1 \rightarrow 2$. This change manifests itself in $\Delta \epsilon^{\mathrm{BNP}}$ of Eq. (4) and may explain the discontinuity around $\hbar \omega_{c}^{0} \simeq 0.5 \hbar \omega_{\text {LO }}$ (marked with a solid arrow in Fig. 2): if we calculate $\Delta \epsilon^{\mathrm{BNP}}+\Delta \epsilon^{\mathrm{pol}}$ from our analysis, assuming that for $\hbar \omega_{c}^{0} / \hbar \omega_{\mathrm{LO}}<0.5$ transitions from the first Landau level to the second take place, we can explain this discontinuity (cf. low-field curves in Fig. 5). For lower energies, still higher values of $n$ would have to be assumed, but for the actual electron density of our heterojunction, the $n=1$ Landau level is expected to be com- 
pletely filled only for $\hbar \omega_{c}^{0} / \hbar \omega_{\mathrm{LO}}<0.2$, and therefore, the assumption of the existence of $1 \rightarrow 2$ transitions at $\hbar \omega_{c}^{0} / \hbar \omega_{\text {LO }} \simeq 0.5$ does not seem to be a reasonable explanation.

It is possible that the discontinuity in the data reflects a discontinuity in the screening as a function of the magnetic field. The dc Hall resistance of our heterojunction clearly shows spin-resolved Hall plateaus and the filling factor $v=n_{e} h / e B$ changes from $v=2$ to $v=1$ just around the observed discontinuity at $\hbar \omega_{c}^{0} \simeq 0.5 \hbar \omega_{\text {LO }}$. Using our model, the discontinuity would be reproduced if for some reason the screening becomes suppressed to $\gamma \simeq 0.5$ at low fields when $v>1$.

At even lower fields, the data cannot be properly described this way. Very recently, Schlesinger, Allen, Hwang, Platzman, and Tzoar reported a shift and a splitting of the CR absorption line in many AlGaAs-GaAs samples with different electron sheet densities and found anomalies at energies described by a universal function of the electron density. ${ }^{27}$ For $n_{e} \simeq 4.0 \times 10^{11} \mathrm{~cm}^{-2}$ the anomaly would be around $\hbar \omega_{c}^{0} \simeq 0.21 \hbar \omega_{\text {LO }}$. We suggest that the anomaly at $\hbar \omega_{c}^{0} \simeq 0.24 \hbar \omega_{\text {LO }}$ (marked with a dashed arrow in Fig. 2) is just this phenomenon. The data at very low fields show an enhancement of the effective mass, which is certainly not caused by polaron effects.

\section{Comparison of $2 \mathrm{D}$ and $3 \mathrm{D}$ resonant polaron effects}

The 2D and 3D systems we have studied both concern the conduction electrons and phonons of the GaAs host material. Therefore, the same material constants, such as $m_{0}^{*}, \alpha$, and $\hbar \omega_{\mathrm{LO}}$, have to be used both for the $2 \mathrm{D}$ electron gas in AlGaAs-GaAs heterojunctions and for the 3D electron gas in $n$-GaAs. The conduction band in GaAs is nonparabolic, and this BNP can be calculated in both cases, using Eq. (4); however, the kinetic energy term $K$ in Eq. (4) depends on the electron density and vanishes in the case of $n$-GaAs. At low fields, several anomalies are present in the CR energies of the $2 \mathrm{D}$ system. At high fields, only Landau-level transitions $0 \rightarrow 1$ take place and the picture seems to be much simpler. Therefore, the high-field data of the $3 \mathrm{D}$ and $2 \mathrm{D}$ systems can be compared directly.

Apart from the contribution to the Landau-level transition energies $\hbar \omega_{c}^{0}$ and $\Delta \epsilon^{\mathrm{BNP}}$, we found that in the lowfield limit the polaron contribution for the 3D $n$-GaAs system is well described by the expression for $\Delta \epsilon^{\mathrm{RSPT}}$. For the case of 2D AlGaAs-GaAs heterojunctions, the screening suppressed any low-field polaron effects, leading to $\Delta \epsilon^{\mathrm{RSPT}} \simeq 0$ in the $2 \mathrm{D}$ case. Recent experiments on an A1GaAs-GaAs heterojunction of about the same mobility confirmed our result that at low fields no mass enhancement due to polaron effects can be observed. ${ }^{28}$ Figure 6 shows a comparison of the relevant resonant polaron correction $\epsilon-\hbar \omega_{c}^{0}-\Delta \epsilon^{\mathrm{BNP}}-\Delta \epsilon^{\mathrm{RSPT}}$ for the high-field data $(0 \rightarrow 1$ transitions) of the $2 \mathrm{D}$ and $3 \mathrm{D}$ systems as a function of the energy of the incident radiation $\epsilon=h v$. Although the resonant polaron effect in 2D AlGaAsGaAs is suppressed by screening, it is still of about the same order of magnitude as the resonant polaron effect in $n$-GaAs.

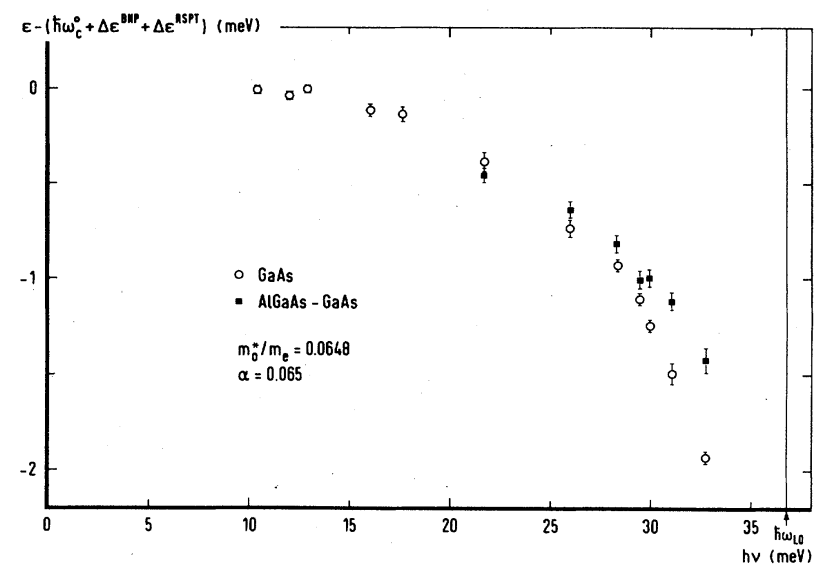

FIG. 6. Comparison of the resonant polaron contribution at high energies for 3D bulk $n-G a A s(O)$ and $2 \mathrm{D}$ AlGaAs-GaAs heterojunctions ( $\boldsymbol{\square})$ as a function of the energy of incident radiation $h v$. The data represent the measured CR transition energy minus the calculated zero-order transition energy $\hbar \omega_{c}^{0}$ and BNP correction $\Delta \epsilon^{\mathrm{BNP}}$, evaluated for the Landau-level transition $0 \rightarrow 1$ using $m_{0}^{*} / m_{e}=0.0648$. For $n$-GaAs, also the low-field polaron correction $\Delta \epsilon^{\mathrm{RSPT}}$ was subtracted, using a coupling constant $\alpha=0.065$; for the 2D AlGaAs-GaAs system this last correction is very small and can be neglected.

\section{CONCLUSION}

We present CR data of the 3D bulk system $n$-GaAs and of the 2D electron system of AlGaAs-GaAs heterojunctions, measured up to energies very close to the energy of the optical phonon. Polaron effects are found to be significant in both systems, and the Landau-level transitions are analyzed with a model that includes nonparabolicity of the conduction band and that evaluates polaron effects from a perturbation scheme of the Fröhlich Hamiltonian.

In a detailed comparison of the model with the experimental 3D CR data we find for the effective mass in $n$ GaAs a value $m_{0}^{*}=0.0648 m_{e}$, and a coupling constant $\alpha=0.065$. We show that in order to obtain the proper limit $\hbar \omega_{\text {LO }}$ for the transition energy, when strong hybridization with the optical phonon has taken place (resonance), band nonparabolicity has to be taken explicitly into account within the relevant polaron model.

The polaron effect is found to be weaker in the 2D electron gas of an AlGaAs-GaAs heterojunction than in a 3D bulk $n$-GaAs crystal. We can account for the magnitude of the measured 2D polaron effects within the framework of the usual polaron model by a nonzero extension of the quasi-two-dimensional electron wave function in the direction perpendicular to the interface and, foremost, by the strength of the screening of the polarization field of the optical phonon due to electron-electron interaction in a Thomas-Fermi approximation. From a fit of our expression for the Landau-level transition energies in the $2 \mathrm{D}$ case (including the suppression of polaron effects due to screening) to the experimental data, it appears that the actual screening is about 1.5 to 2 times as strong as estimated from the Thomas-Fermi model, and we find that with such a strong screening polaron effects at low fields will be suppressed very strongly. The anomalies observed at 
low fields in AlGaAs-GaAs are not yet understood, but they do not seem to be due to polaron effects. We hope that the availability of CR data close to the resonance will stimulate theoretical efforts to extend the detailed calculation of Das Sarma and Mason ${ }^{22}$ over the whole energy range.

\section{ACKNOWLEDGMENTS}

We are pleased to thank Dr. J. Wolter and Dr. T. van Oirschot of the Philips' Research Laboratories for provid- ing the AlGaAs-GaAs heterojunctions and the $n$-GaAs sample. We are grateful to Dr. S. Das Sarma for communicating theoretical results ${ }^{22}$ prior to publication. We would also like to thank Dr. U. Merkt for sending us results of recent work, ${ }^{28}$ where experiments similar to ours are discussed. Part of this work has been supported by the "Stichting voor Fundamenteel Onderzoek der Materie" (FOM) with financial support of the "Nederlandse Organisatie voor Zuiver Wetenschappelijk Onderzoek" (ZWO).
${ }^{1}$ D. M. Larsen, Proceedings of the 10th International Conference on the Physics of Semiconductors, Cambridge, Mass., 1970, edited by S. P. Keller, J. C. Hensel, and F. Stern (U.S. AEC, Washington, D.C., 1970), p. 145.

${ }^{2}$ For a review see Polarons in Ionic Crystals and Polar Semiconductors, edited by J. T. Devreese (North-Holland, London, 1972).

${ }^{3}$ T. Ando, A. B. Fowler, and F. Stern, Rev. Mod. Phys. 54, 437 (1982).

${ }^{4}$ For a review, see Proceedings of the 5th International Conference on Electronic Properties of Two-Dimensional Systems, Oxford U.K., 1983, edited by R. J. Nicholas, Surf. Sci. 142 (1984).

${ }^{5}$ M. Horst, U. Merkt, and J. P. Kotthaus, Phys. Rev. Lett. 50, 754 (1983).

${ }^{6}$ W. Seidenbusch, G. Lindemann, R. Lassnig, J. Edlinger, and G. Gornik, Surf. Sci. 142, 375 (1984).

${ }^{7}$ G. Lindemann, R. Lassnig, W. Seidenbusch, and E. Gornik, Phys. Rev. B 28, 4693 (1983).

${ }^{8}$ H. Sigg, H. J. A. Bluyssen, and P. Wyder, Solid State Commun. 48, 897 (1983).

${ }^{9}$ S. Das Sarma, Phys. Rev. B 27, 2590 (1983).

${ }^{10}$ X. X. Liang and S. W. Gu, Solid State Commun. 50, 505 (1984).

${ }^{11}$ H. Sigg, H. J. A. Bluyssen, and P. Wyder, IEEE J. Quantum Electron. QE-20, 616 (1984).

${ }^{12}$ A. Mooradian and G. B. Wright, Solid State Commun. 4, 431 (1966).

${ }^{13}$ K. van Hulst, C. J. M. Aarts, A. R. de Vroomen, and P.
Wyder, J. Magn. Magn. Mater. 11, 317 (1979).

${ }^{14}$ R. E. Horstman, E. J. v.d. Broek, J. Wolter, R. W. van der Heijden, G. L. J. A. Rikken, H. Sigg, P. M. Frijlink, J. Maluenda, and J. Hallais, Solid State Commun. 50, 753 (1984).

${ }^{15}$ D. M. Larsen, Ref. 2, p. 237.

${ }^{16}$ S. Das Sarma, Phys. Rev. Lett. 52, 859 (1984).

${ }^{17}$ Y. B. Levinson and E. I. Rahsba, Rep. Prog. Phys. 36, 1499 (1973).

${ }^{18}$ This result can also be evaluated from the polaron energy expression of Ref. 16 where, however, an erroneous prefactor of $1 / \sqrt{2}$ should be left out; S. Das Sarma (private communication).

${ }^{19}$ R. Lassnig and W. Zawadzki, Surf. Sci. 142, 388 (1984).

${ }^{20}$ F. Stern and W. E. Howard, Phys. Rev. 163, 816 (1967).

${ }^{21}$ P. J. Price, J. Vac. Sci. Technol. 19, 599 (1981).

${ }^{22}$ S. Das Sarma and B. A. Mason (unpublished).

${ }^{23}$ D. L. Rode, Phys. Rev. B 2, 1012 (1970).

${ }^{24}$ G. E. Stillman, D. M. Larsen, C. M. Wolfe, and R. C. Brandt, Solid State Commun. 9, 2245 (1971).

${ }^{25}$ D. M. Larsen, Proceedings of the 17 th International Conference on the Physics of Semiconductors, San Francisco, 1984 (unpublished).

${ }^{26}$ F. Stern, Phys. Rev. Lett. 18, 546 (1967).

${ }^{27}$ Z. Schlesinger, S. J. Allen, J. C. M. Hwang, P. M. Platzman, and N. Tzoar, Phys. Rev. B 30, 435 (1984).

${ }^{28}$ M. Horst, U. Merkt, W. Zawadzki, J. C. Maan, and K. Ploog, Solid State Commun. 53, 403 (1985). 